\title{
Multisensor fusion for the accurate classification of vegetation in complex ecosystems
}

\author{
Javier Marcello \\ Institute of Oceanography and Global Change (IOCAG) \\ University of Las Palmas de Gran Canaria (ULPGC) \\ Unidad Asociada ULPGC - CSIC, \\ Las Palmas de Gran Canaria, Spain \\ javier.marcello@ulpgc.es \\ Edurne Ibarrola-Ulzurrun \\ Institute of Oceanography and Global Change (IOCAG) \\ University of Las Palmas de Gran Canaria (ULPGC) \\ Las Palmas de Gran Canaria, Spain \\ edurne.ibarrola101@alu.ulpgc.es
}

\author{
Dionisio Rodríguez-Esparragón \\ Institute of Oceanography and Global Change (IOCAG) \\ University of Las Palmas de Gran Canaria (ULPGC) \\ Unidad Asociada ULPGC - CSIC, \\ Las Palmas de Gran Canaria, Spain \\ dionisio.rodriguez@ulpgc.es \\ Consuelo Gonzalo-Martín \\ Center for Biomedical Technology (CTB) \\ Universidad Politécnica de Madrid (UPM) \\ Madrid, Spain \\ consuelo.gonzalo@ctb.upm.es
}

\begin{abstract}
The use of geospatial tools to monitor natural ecosystems is a fundamental task to preserve the environment. In this context, remote sensing data can provide a valuable source of information to complement field observations, offering frequent and accurate imagery to support the mapping and monitoring of natural areas. The growing availability of hyperspectral (HS) data can provide a valuable solution but the spectral richness provided by hyperspectral sensors is usually at the expense of spatial resolution. To alleviate this inconvenience, instead of satellite platforms, airborne sensors can be considered. In this work, the accurate mapping of a complex shrubland ecosystem has been accomplished using multisensor imagery. Specifically, airborne CASI data ( 68 bands and $75 \mathrm{~cm}$ of pixel size) has been fused with an orthophoto $(25 \mathrm{~cm})$ to increase the spatial detail. A comprehensive analysis of 11 sharpening algorithms has been performed and, to improve the Support Vector Machine (SVM) classification accuracy, different input features have been considered. Excellent results have been achieved and the importance to improve the spatial resolution has been demonstrated.
\end{abstract}

Keywords-remote sensing, hyperspectral, sharpening, classification, ecosystems.

\section{INTRODUCTION}

Natural areas provide useful services to enhance the human well-being. Nevertheless, in recent years, there has been a degradation of ecosystems and biodiversity, due to increase of tourism, agricultural land use, etc. Therefore, monitoring the state of conservation of natural areas is important to select the appropriate decisions and policies. Insitu observations have been the main data source to assess the status of ecosystems, however this procedure is laborintensive, time-consuming and, hence, more difficult to be frequently repeated. In this sense, remote sensing imagery can provide valuable data and supplement field observations, offering a synoptic view and consistent information in space and time. Thus, remote sensing is a repeatable and accurate tool to support the mapping and monitoring of ecosystems [1]. In this context, due to the important progress that sensors have experience in last decades, remote sensing can supply a considerable amount of data with high spectral, spatial and temporal resolutions. In consequence, it is a cost-effective strategy for environmental monitoring, especially for large natural areas or when frequent information is required.

Mapping of broad habitat types with remote sensing is an accepted practice but it is, generally, performed at coarse scales. Unfortunately, such coarse spatial detail is not sufficient for the monitoring of complex areas. Specifically, insular areas include, in general, smaller and heterogeneous ecosystems. Therefore, these areas need imagery with the maximum spatial and spectral resolutions. In this sense, the recent availability of new sensors on board satellites and airborne platforms, with better spatial and spectral capabilities, can facilitate the generation of accurate vegetation maps in natural areas. Unfortunately, optimum simultaneous resolutions cannot, generally, be achieved because, for technological reasons. There is a compromise between the signal-to-noise received, the spectral bandwidth and the spatial field of view provided by the sensor. For example, very high spatial resolution satellite data usually have moderate spectral resolution and, conversely, hyperspectral (HS) sensors cannot provide imagery with fine spatial resolution. To solve the previous inconvenience, airborne hyperspectral instruments can provide higher spatial detail but there is still a trade-off between both resolutions to get an acceptable signal-to-noise ratio. To overcome this inconvenience, different HS sharpening and fusion techniques have been developed for many Earth observation applications [2-6].

Fusion techniques combine imagery from different sources to generate a new image with higher quality than the original images, providing more reliable and detailed information. For high resolution satellite sensors, pansharpening (fusion at pixel level) increases the spatial resolution of the multispectral channels using a high resolution panchromatic band. With the appearance of HS instruments, new sharpening methods have been developed. HS sharpening is an important research topic and, so far, a reduced number of comparative studies have been presented in the literature. For example, [2] and [4] include a comparative analysis using techniques developed in the last decade. In this sense, one important contribution of this work is to assess different HS sharpening techniques but in the context of monitoring complex ecosystems.

The key advantage of hyperspectral data, compared to multispectral, is the considerably greater amount of spectral information, which benefits the reliability, accuracy and information extraction capability in complex studies, such as vegetation mapping in challenging ecosystems. However, HS sharpening is more difficult than multispectral pansharpening due to the high dimensionality of HS imagery. Therefore, the challenge is to develop simple but reliable HS fusion models. 
Another active area in the image processing field is the development of classification methodologies. In particular, the classification of remote sensing hyperspectral data is challenging due to the high dimensionality of the data, the spectral redundancy and the common lack of detailed training datasets. For this reason, specific approaches have been developed, like SVM, random forests, logistic regressions or deep learning [7]. Unmixing techniques have also been considered with HS data to separate the pixel spectra into a collection of endmembers (pure spectral signatures) and their corresponding abundances [8]

The main objective of this work focusses on the generation of challenging vegetation maps, at species level, in a complex shrubland ecosystem, with mixed vegetation and small size plants, using remote sensing data. Hyperspectral airborne imagery has been used and a methodological framework for classification is presented for the monitoring and management of similar natural protected areas.

\section{DATASETS AND Methodology}

\section{A. Area of Study}

Canary Islands, in Spain, are one of the most remarkable biodiversity hotspots on the Earth. This archipelago includes important endemic flora highly vulnerable to environmental changes. For this reason, almost half of the total area is protected to preserve this vegetation richness and singularity.

Specifically, the Teide National Park (Tenerife Island) is one of the most important natural areas of Canary Islands and Spain. The protected area covers 18,990 ha and it is made by the Teide peak $(3718 \mathrm{~m})$ and a giant crater at an altitude higher than $2000 \mathrm{~m}$ over the sea level. The extreme climate is mainly determined by the elevation and orientation respect to the Sun and Trade winds. The vegetation of the Teide Park responds to thermic and hydric stress with a shrub physiognomy.

In the last decades, the area has experienced important changes in its vegetal resources. Apart from the important touristic pressure, mainly, large populations of rabbits, as well as drought episodes, have led to a change in the vegetation structure. In this context, the species of major interest for the Park managers (Fig. 1) are Spartocytisus supranubius, Descurainia bourgaeana, Pterocephalus lasiospermus and Pinus canariensis.

\section{B. Remote Sensing Imagery}

In order to better discriminate similar species, HS data has been considered. Specifically, a flight in the Teide National Park (Tenerife, Spain) was made on June 1, 2017, using the INTA CASA C-2012-200 aircraft equipped with the CASI 1500i push-broom hyperspectral sensor. CASI records radiance in the $380-1050 \mathrm{~nm}$ spectral range using 14 bits per sample. It can be configured with a maximum of 288 channels but in our case, to achieve a fine pixel size of 75 $\mathrm{cm}$, only 68 bands were selected to increase the signal-tonoise ratio [9].

Even though CASI HS data was available with high spatial resolution, due to the small size of some vegetal species, an orthophoto of the same month was used to increase the spatial detail.

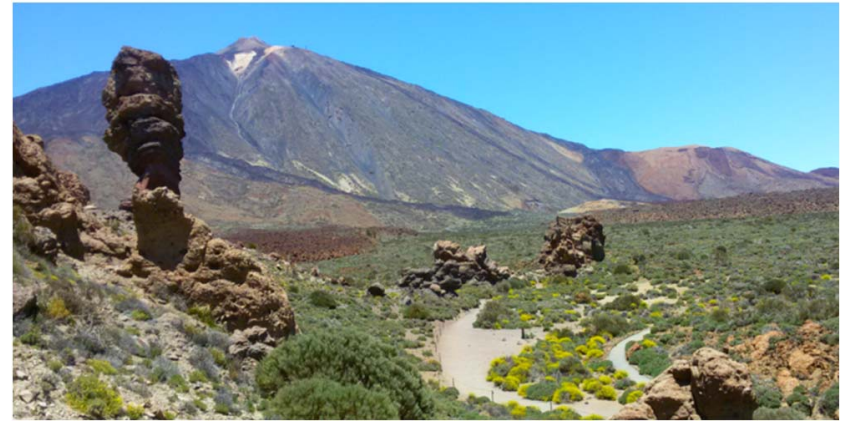

(a)

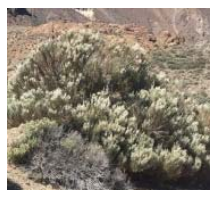

(b)

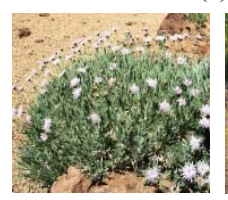

(c)

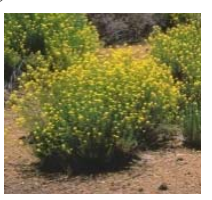

(d)

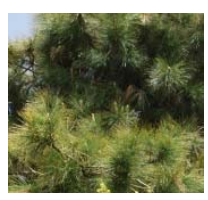

(e)
Fig. 1. (a) Teide National Park. Vegetation species: (b) Spartocytisus supranubius, (c) Pterocephalus lasiospermus, (c) Descurainia bourgaeana, and (d) Pinus canariensis.

Specifically, a 3 true color bands orthoexpress product with $25 \mathrm{~cm}$ of ground sample distance was acquired from GRAFCAN [10]. Hence, the CASI image was sharpened using the orthophoto. Fig. 2 shows both datasets.

\section{Hyperspectral Sharpening}

Preliminary preprocessing algorithms were applied to the CASI image. Specifically, geometric, radiometric and ATCOR-4 atmospheric corrections were done [9]. Next, CASI data and the orthophoto were co-registered using a large set of distributed and representative ground control points before the sharpening process could be applied between the hyperspectral image and the intensity value of the orthophoto.

Pansharpening techniques can be practically divided into component substitution (CS), multiresolution analysis (MRA), Bayesian, hybrid and deep learning methods [11]. HS sharpening can be considered a special case of the multispectral fusion problem [4]. A total of 11 sharpening algorithms of different categories have been chosen for the assessment [2]. The algorithms selected from the CS family are Principal Component Analysis (PCA), Gram-Schmidt (GS) and GS Adaptive (GSA). From the MRA family, the Smoothing Filter-based Intensity Modulation (SFIM), the Modulation Transfer Function - Generalized Laplacian Pyramid (MTF-GLP) and the MTF-GLP with High Pass Modulation (MTF-GLP-HPM) have been analyzed. Regarding the Bayesian methods, the Naive, the Sparse and the HySure approaches were included. Finally, from the hybrid category, Guided Filter Principal Components Analysis (GFPCA) and Coupled Nonnegative Matrix Factorization (CNMF) were selected.

To evaluate the quality of the HS fused images, visual and quantitative approaches were used [12]. In the visual assessment, the spectral fidelity and the spatial enhancement were compared. Also, the following quality metrics were used to measure the spatial and the spectral quality: spectral cross correlation (CC), spectral angle mapper (SAM), root mean squared error (RMSE), the erreur relative globale adimensionnelle de synthèse (ERGAS) and the spatial cross correlation (sCC). Finally, classification was also considered to indirectly validate the quality of the fused images. 


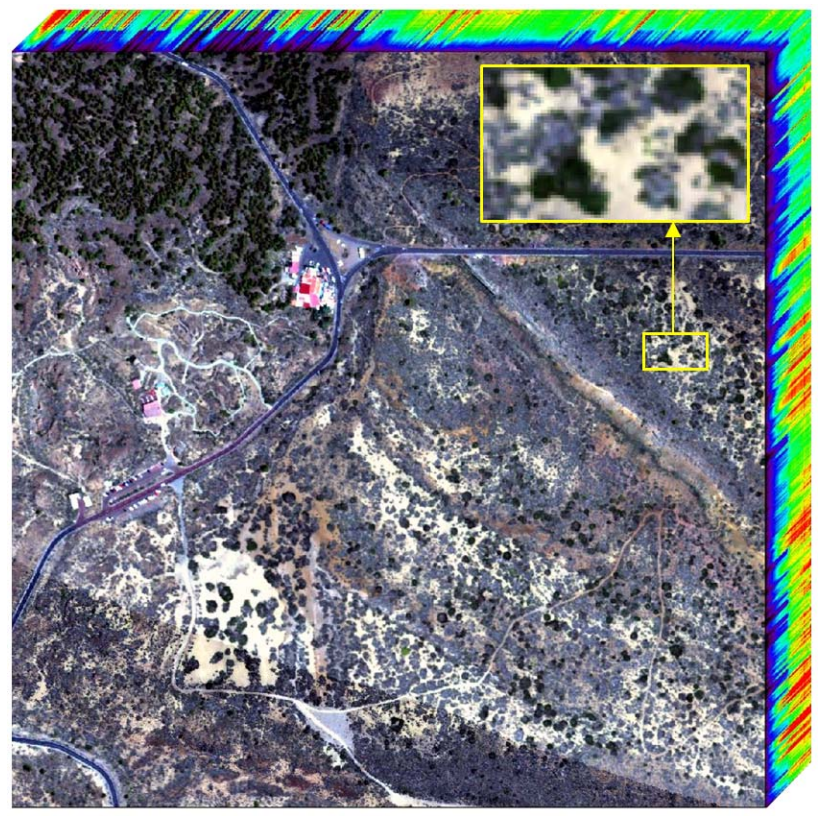

(a)

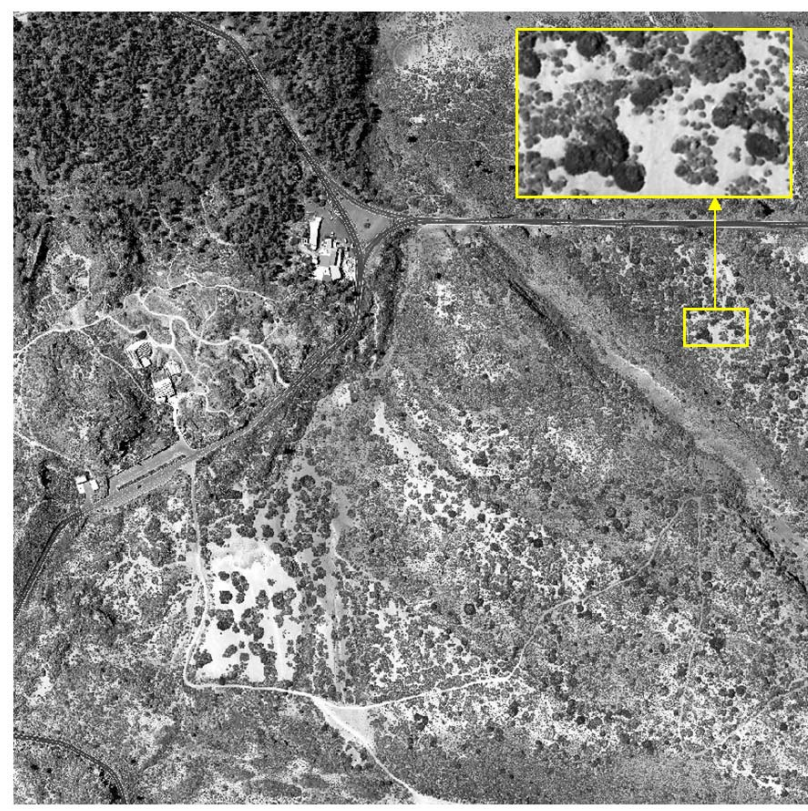

(b)

Fig. 2. Remote sensing imagery: (a) CASI hyperspectral original cube (true color composite in the frontal view), and (b) Orthophoto intensity.

\section{Hyperspectral Classification}

The corrected and sharpened HS image using the best method was input to the classification stage. However, a preliminary feature extraction step was included to analyze the potential improvement in the mapping accuracy when considering additional information.

In this context, the features analyzed were the optimal components after the application of Principal Component Analysis (PCA) and Minimum Noise Fraction (MNF) transforms to check the performance with few bands to avoid the Hughes phenomenon [13]. Also, texture information derived from the Gray-Level-Co-occurrence Matrix applied to the first principal component was considered. After a preliminary assessment of the texture parameters, and to avoid the inclusion of redundant information, only the variance, homogeneity and dissimilarity parameters were finally used [14]. Finally, the Normalized Difference
Vegetation Index (NDVI) was computed. NDVI quantifies vegetation by measuring the normalized difference between near-infrared (which vegetation strongly reflects) and red light (which vegetation absorbs) spectral bands.

Finally, the spectral bands and the resulting features (PCA and MNF components, texture bands and abundance maps) were used as input data to the classifier.

Supervised classification methods learn from a training dataset and assign the appropriate classes to the remaining pixels of the image. Efficient classifiers used in remote sensing are Maximum Likelihood classification, Random Forest, Support Vector Machines. On the other hand, deep learning algorithms are starting to become popular into the remote sensing community and have proven to be efficient in extracting land cover classes. However, due to the amount of training data required, they are not practical in many scenarios and when using a large numbers of bands [15].

In this work, SVM has been selected due to its excellent good performance and robustness with HS data, even when using a small training database $[16,17]$. Also, a recent work comparing SVM, RF, deep convolutional neural networks, sparse representation-based classifiers and logistic regression techniques, demonstrated that SVM is widely used because of its stability, accuracy and simplicity [7]. The Gaussian radial basis function kernel was selected and the corresponding parameters were properly adjusted [18].

The classification performance was quantitatively compared using an independent dataset of test regions and computing the confusion matrix and its derived measures (overall accuracy and kappa coefficient).

\section{RESULTS}

In the next section, the performance of the HS sharpening algorithms is presented. Next, the classification results using the additional feature information is included.

\section{A. Hyperspectral Sharpening}

Fig. 3 shows the sharpening results for a small area. The spectral distortion introduced by each sharpening algorithm can be appreciated. In order to better analyze the spatial enhancement, a small section is included in Fig. 4. The resolution ratio is 3 and a great improvement in the spatial detail is evident in some fused imagery when compared with the original CASI (Fig 3(a)). Specifically, the best sharpening algorithms at visual level are GS, GSA and PCA; however, in accordance with the trade-off of the sharpening techniques between spatial improvement and spectral degradation, it can be clearly appreciated in the road that these component substitution methods degrade in a greater extent the spectral fidelity. On the other hand, other algorithms like SFIM, MTFs and GFPCA better preserve the original spectral information but the spatial improvement is not as good. Finally, the remaining methods (CNMF, Bayes and HySure) are not appropriate in this fusion scenario. Moreover, these techniques imply higher computation times.

Quality indices (Table I) corroborate the visual perception, providing precise information to compare the fusion algorithms. Green colors highlight the best techniques while red ones the worst methods. In general, the best spectral quality is achieved by SFIM and MFT_GLP. GS and PCA provide the best spatial quality (sCC). HySure is not properly working in this situation. 


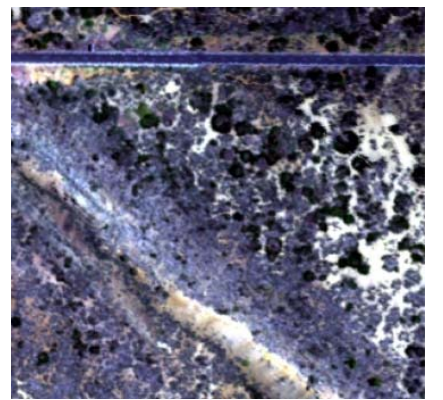

(a)

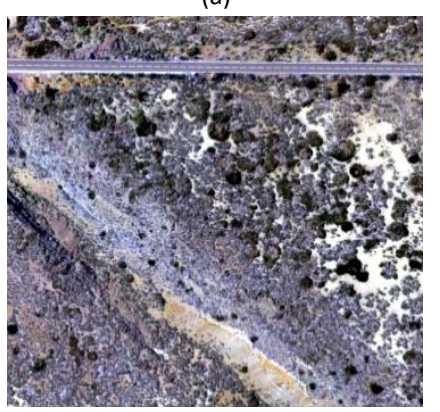

(e)

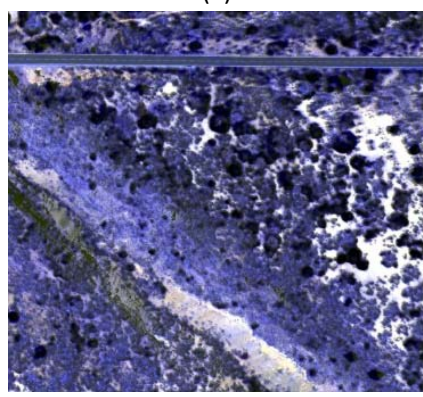

(i)

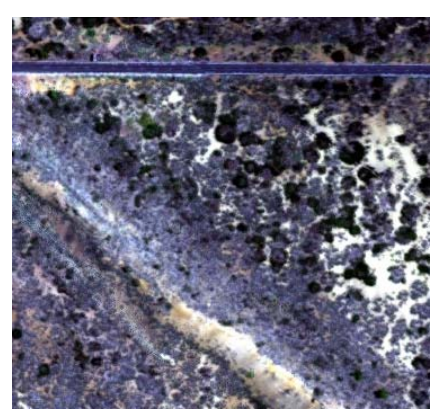

(b)

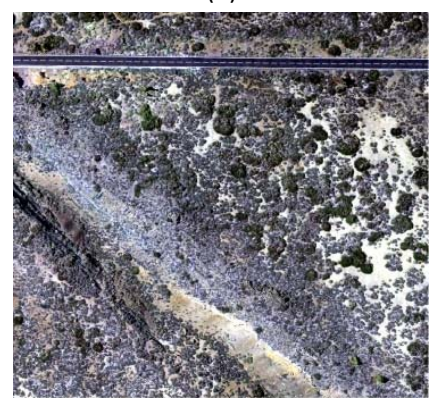

(f)

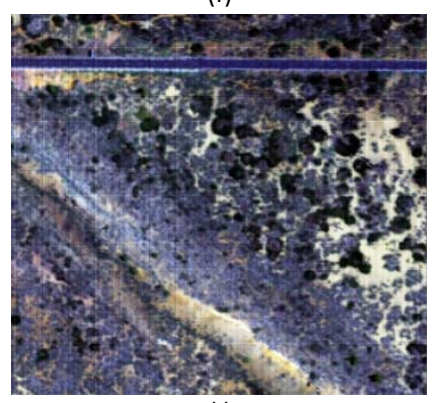

(j)

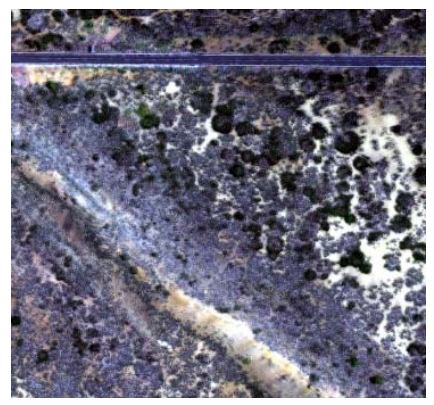

(c)

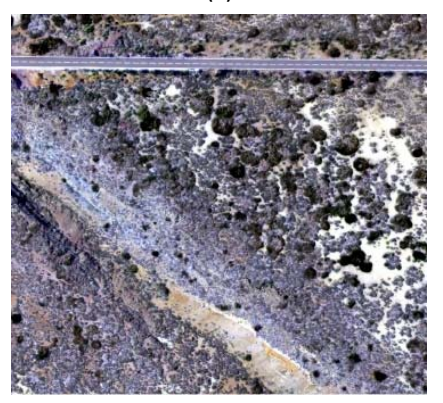

(g)

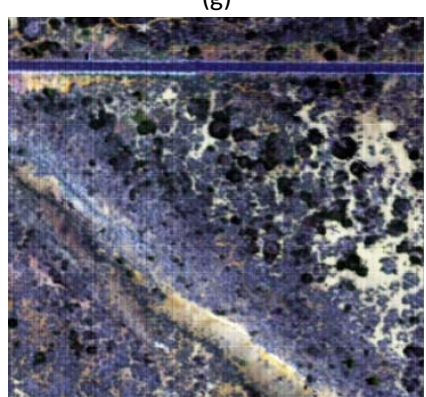

(k)

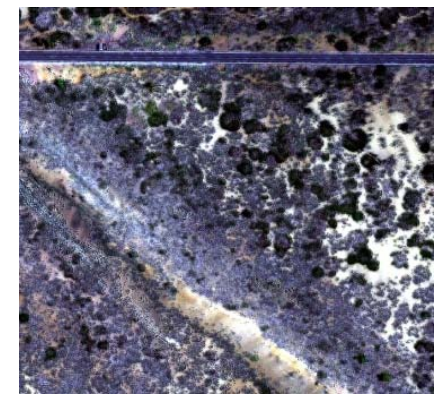

(d)

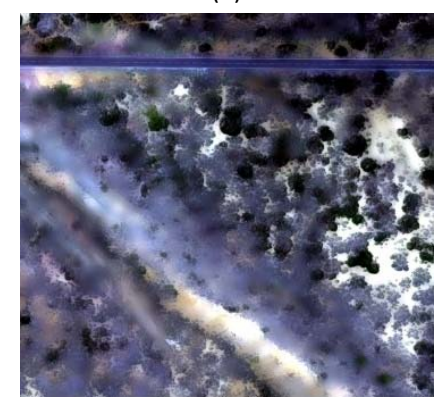

(h)

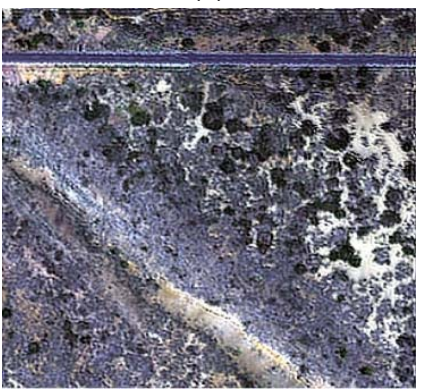

(I)

Fig. 3. (a) HS original, (b) SFIM, (c) MTF_GLP, (d) MTF_GLP_HPM, (e) GS, (f) GSA, (g) PCA, (h) GFPCA, (i) CNMF, (j) Bayes Naive, (k) Bayes Sparse, and (1) HySure.

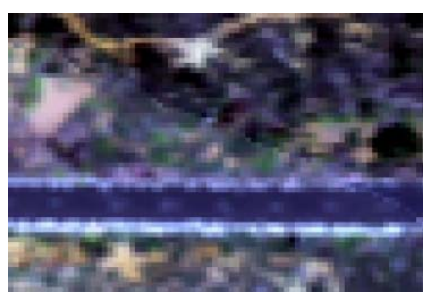

(a)

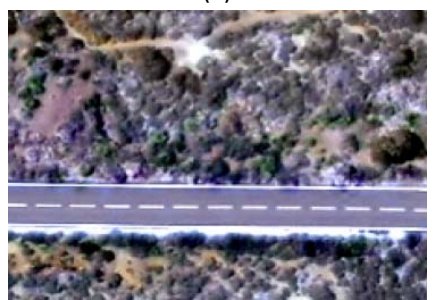

(e)

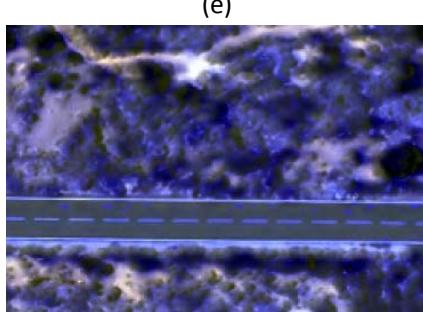

(i)

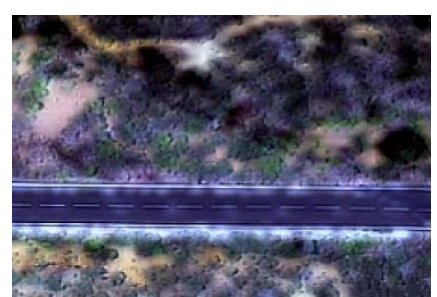

(b)

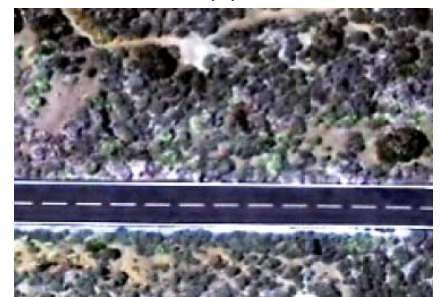

(f)

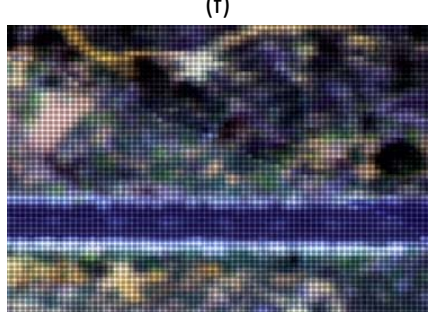

(j)

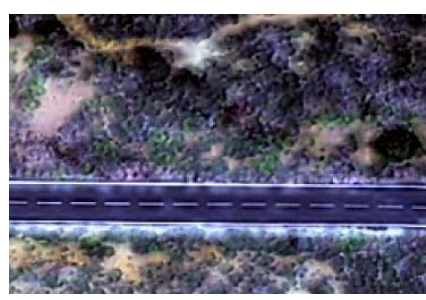

(c)

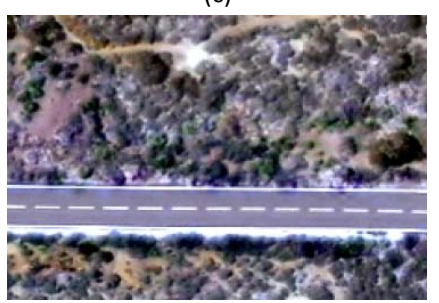

(g)

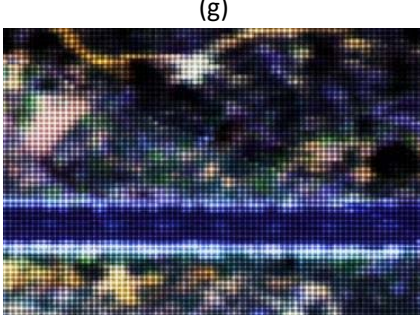

(k)

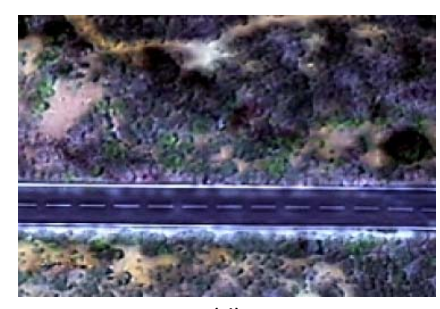

(d)

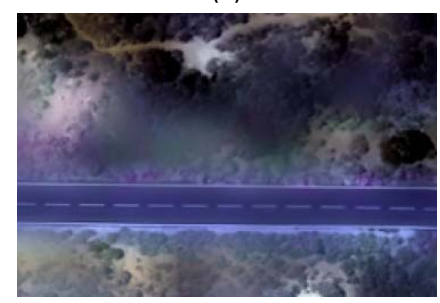

(h)

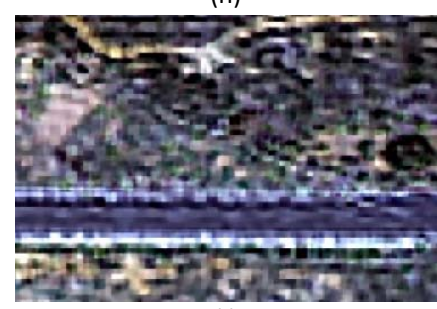

(I)

Fig. 4. (a) HS original, (b) SFIM, (c) MTF_GLP, (d) MTF_GLP_HPM, (e) GS, (f) GSA, (g) PCA, (h) GFPCA, (i) CNMF, (j) Bayes Naive, (k) Bayes Sparse, and (1) HySure. 
TABLE I

PERFORMANCE OF THE SHARPENING ALGORITHMS FOR THE FUSION OF CASI DATA AND THE ORTHOPHOTO.

\begin{tabular}{l|ccccc|cc}
\hline \multirow{2}{*}{ Method } & \multicolumn{5}{c|}{ Quality metrics } & \multicolumn{2}{c}{ Classification } \\
\cline { 2 - 8 } & CC & SAM & RMSE & ERGAS & sCC & OA (\%) & Kappa \\
\hline SFIM & 0.946 & 0.75 & 100.3 & 9.06 & 0.873 & 89.2 & 0.87 \\
MTF_GLP & 0.920 & 1.08 & 140.5 & 4.05 & 0.970 & 88.7 & 0.86 \\
MTF_GLP_HPM & 0.902 & 1.13 & 147.3 & 11.22 & 0.918 & 90.4 & 0.89 \\
GS & 0.628 & 2.33 & 290.4 & 7.59 & 0.984 & 96.3 & 0.96 \\
GSA & 0.773 & 4.81 & 277.7 & 8.98 & 0.980 & 94.7 & 0.94 \\
PCA & 0.618 & 2.24 & 297.2 & 7.61 & 0.983 & 95.6 & 0.95 \\
GFPCA & 0.878 & 2.69 & 159.2 & 4.61 & 0.622 & 83.5 & 0.80 \\
CNMF & 0.710 & 2.55 & 228.7 & 6.47 & 0.753 & 82.4 & 0.79 \\
Bayes Naive & 0.771 & 3.92 & 293.4 & 46.02 & 0.088 & 86.1 & 0.84 \\
Bayes Sparse & 0.800 & 4.15 & 254.0 & 36.26 & 0.094 & 85.4 & 0.83 \\
HySure & 0.765 & 4.45 & 302.0 & 8.02 & 0.269 & 76.3 & 0.72 \\
\hline
\end{tabular}

As indicated, another quantitative indicator to compare the HS sharpening methods was the accuracy of the SVM maps generated by each algorithm using the same parameters and training regions. The last 2 columns of Table I provide the overall accuracy and the kappa values. As a reference, the overall accuracies for the original input images (CASI HS and the true-color orthophoto) are: $80.53 \%$ and $75.03 \%$, respectively. Therefore, an important improvement is achieved when selecting the most appropriate sharpening algorithms. As indicated, due to the complexity of the ecosystem analyzed and the great importance of the spatial information, related to the small size of the vegetation, the highest accuracy is provided by GS and PCA, improving in more than $15 \%$ the overall accuracy with respect to the original hyperspectral CASI image.

\section{B. Hyperspectral Classification}

Once the best sharpening algorithm is identified (GS), to further improve the mapping results, additional information has been considered. In particular, the following combinations were assessed when included as input to the SVM classifier:

- Inclusion of the vegetation index (NDVI).

- Inclusion of texture parameters (variance, homogeneity and dissimilarity).

- Inclusion of both previous features.

- Inclusion of 4 PCA components. PCA was applied to the hyperspectral fused data (CASI_GS) and the first bands providing useful information were selected.

- Inclusion of 9 MNF components. As for PCA, MNF was applied to the HS fused data (CASI_GS) and the first bands providing useful information were selected.

The results are presented in Table II, where a slight improvement in the mapping accuracy is appreciated when including additional textural information. On the other hand, using very few bands after the PCA or MNF transformations, good accuracy is also obtained.

Fig. 5 shows examples of different vegetation maps for the area of Fig. 3 and including, as well, a zoom to better appreciate the spatial information. The map achieving the best accuracy has been included and, as a reference, also the SVM maps for the original orthophoto, the original CASI image and the CASI fused with the GS algorithm. As the most important information are the vegetation species, land has been set to white and transparency has been applied to the road and shadows. The colors selected for the vegetation are: green for Spartocytisus supranubius, thistle for Pterocephalus lasiospermus, yellow for Descurainia bourgaeana and dark green for Pinus canariensis.

The 3-band orthophoto, even having excellent spatial resolution, is not able to properly discriminate the vegetation classes. Hyperspectral data provides much more information to better classify the different plant species but a clear spatial improvement is evident thanks to the GS sharpening.

\section{CONCLUSIONS}

This work addresses the mapping of complex and vulnerable shrubland ecosystems using hyperspectral imagery. The Teide National Park has been selected for the study as it is made up of small and mixed vegetation, but the conclusions derived can be applicable to similar zones.

As the original HS data does not provide the optimum spatial resolution to map the small plants to be monitored, a thorough analysis of 11 HS sharpening algorithms has been completed to assess the HS fusion performance using visual and quantitative approaches. Specifically, airborne CASI data, with 68 bands and $75 \mathrm{~cm}$ of pixel size, has been used and it has been fused with an orthophoto with higher spatial resolution $(25 \mathrm{~cm})$.

In general, the comparison using the visual assessment and quality indices correlate. The spectral quality indices considered are mostly consistent but show differences in some cases. In addition, classification has also been used to evaluate the sharpening techniques. SFIM and MTFs have demonstrated good spectral preservation but the spatial improvement is not as good, while GS, GSA and PCA properly transfer the spatial detail. As the spatial component is critical in this ecosystem, the best classification results are also provided by these component substitution sharpening algorithms.

Next, an additional analysis was carried out to identify the best feature to improve the final mapping. A minor improvement in the classification accuracy was gained including textural information.

In summary, we have demonstrated the importance of the multiplatform HS sharpening when dealing with heterogeneous shrubland ecosystem where the spatial information is critical and should be well incorporated to generate accurate thematic maps. Further to this work, we are currently analyzing the use of HS instruments onboard drone platforms for the monitoring of challenging ecosystems.

TABLE II

CLASSIFICATION ACCURACY FOR THE DIFFERENT INPUT COMBINATIONS.

\begin{tabular}{l|cc}
\hline \multicolumn{1}{c|}{ Input to the classifier } & \multicolumn{2}{c}{ Classification } \\
\cline { 2 - 3 } & OA (\%) & Kappa \\
\hline CASI_GS (68 bands) & 96.3 & 0.956 \\
CASI_GS + NDVI (69 bands) & 96.3 & 0.956 \\
CASI_GS + Texture (71 bands) & 96.8 & 0.962 \\
CASI_GS + NDVI + Texture (72 bands) & 96.8 & 0.962 \\
PCA[CASI_GS] (4 bands) & 90.9 & 0.893 \\
MNF[CASI_GS] (9 bands) & 90.8 & 0.892 \\
\hline
\end{tabular}




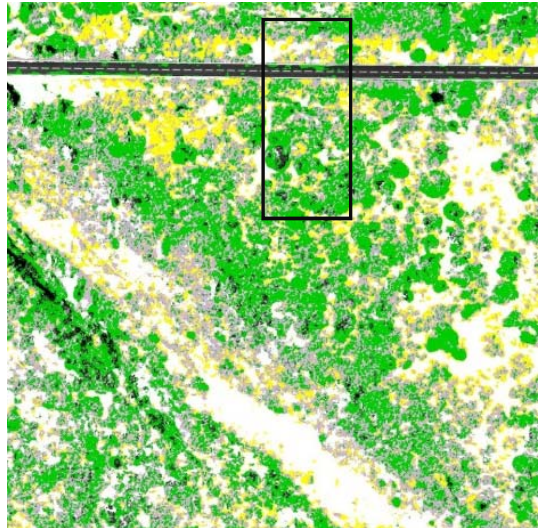

(a)

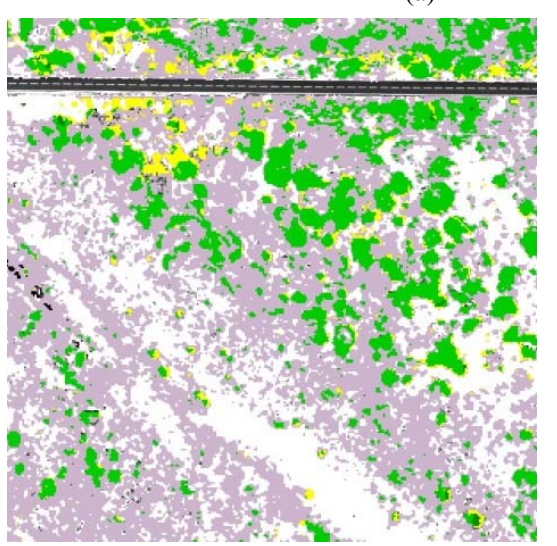

(b)

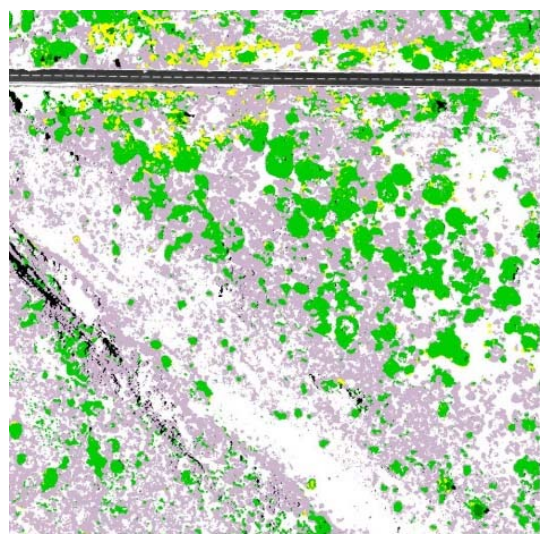

(c)

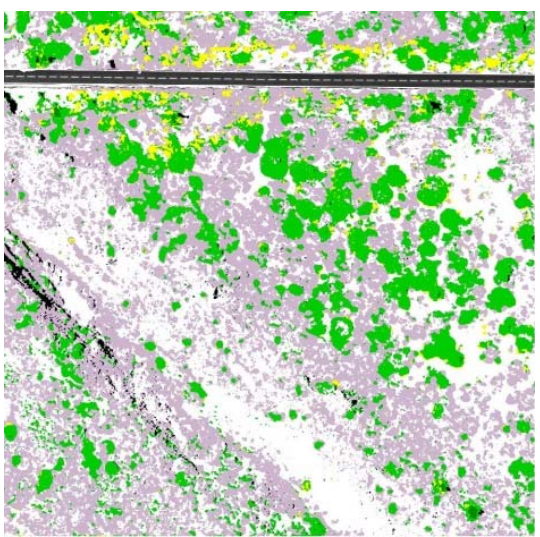

(d)

Fig. 5. SVM maps using the following input data: (a) Orthophoto (RGB bands), (b) CASI original, (c) CASI fused with GS, and (d) CASI fused with GS and including NDVI and texture features.

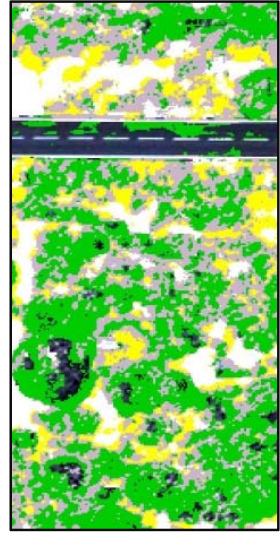

\section{ACKNOWLEDGMENT}

This work has been supported by the ARTEMISAT-2 (CTM2016-77733-R) project, funded by the Spanish Agencia Estatal de Investigación (AEI) and the European Fondo Europeo de Desarrollo Regional (FEDER)

\section{REFERENCES}

[1] C. Corbane, S. Lang, K. Pipkins, S. Alleaume, M. Deshayes, V.E.G. Millán, T. Strasser, J.V. Borre, S. Toon, and F. Michael. "Remote sensing for mapping natural habitats and their conservation statusNew opportunities and challenges," International Journal of Applied Earth Observation and Geoinformation, vol. 37, pp.7-16, 2015

[2] L. Loncan, L. B. Almeida, J. B. Dias, X. Briottet, J. Chanussot, N. Dobigeon, S. Fabre, W. Liao, G. A. Licciardi, M. Simoes, J. Y. Tourneret, M. A. Veganzones, G. Vivone, Q. Wei, and N. Yokoya, "Hyperspectral pansharpening: a review," IEEE Geosciences and Remote Sensing Magazine, vol. 3, no. 3, pp. 27-46, Sep. 2015.

[3] C. Pohl and J. van Genderen, "Structuring contemporary remote sensing image fusion," International Journal of Image and Data Fusion, vol. 6, pp. 3-21, 2015

[4] N. Yokoya, C. Grohnfeldt, and J. Chanussot, "Hyperspectral and multispectral data fusion: A comparative review of the recent literature," IEEE Geoscience and Remote Sensing Magazine, vol. 5, pp. 29-56, 2017.

[5] G. Vivone, L. Alparone, J. Chanussot, M. Dalla Mura, A. Garzelli, G. Licciardi, R. Restaino, and L. Wald, "A critical comparison among pansharpening algorithms," IEEE Transactions on Geoscience and Remote Sensing, vol. 53, no. 5, pp. 2565-2586, 2015.

[6] J. Marcello, A. Medina, and F. Eugenio. "Evaluation of spatial and spectral effectiveness of pixel-level fusion techniques," IEEE Geoscience and Remote Sensing Letters, vol. 10, pp. 432-436, 2013.

[7] P. Ghamisi, J. Plaza, Y. Chen, J. Li, and A. Plaza, "Advanced spectral classifiers for hyperspectral images," IEEE Geosciences and Remote Sensing Magazine, pp. 8-32, march 2017.

[8] J. Bioucas, A. Plaza, N. Dobigeon, M. Parente, Q. Du, P. Gader, and J. Chanussot, "Hyperspectral unmixing overview: Geometrical, statistical, and sparse regression-based approaches," IEEE J. Select. Topics Applied Earth Observation and Remote Sensing, vol. 5, pp. 354-379, 2012.

[9] E. de Miguel, A. Fernández-Renau, E. Prado, M. Jiménez, Ó. G. de la Cámara, C. Linés, et al., "The processing of CASI-1500I data at INTA PAF," in EARSeL eProceedings, vol. 13, pp. 30-37, 2014.

[10] Cartográfica de Canarias S.A. (GRAFCAN). Gobierno de Canarias. https://www.grafcan.es/

[11] J. Yang, Y-Q. Zhao, and J. C-W. Chan, "Hyperspectral and Multispectral Image Fusion via Deep Two-Branches Convolutional Neural Network," Remote Sensing, vol. 10, nº 5, pp. 800, 2018.

[12] L. Wald, T. Ranchin, and M. Mangolini, "Fusion of satellite images of different spatial resolutions: assessing the quality of resulting images," Photogrammetric engineering and remote sensing, vol. 63, pp. 691-699, 1997.

[13] E. Ibarrola-Ulzurrun, J. Marcello, and C. Gonzalo-Martin, "Assessment of Component Selection Strategies in Hyperspectral Imagery," Entropy, vol. 19, no. 12, pp. 666, 2017.

[14] J Marcello, F Eugenio, J Martín, and F Marqués, "Seabed mapping in coastal shallow waters using high resolution multispectral and hyperspectral imagery," Remote Sensing, vol. 10, no. 8, pp.1208, 2018

[15] X. Yu, X. Wu, C. Luo, and P. Ren, "Deep learning in remote sensing scene classification: a data augmentation enhanced convolutional neural network framework," GIScience \& Remote Sensing, vol. 54, no. 5, pp. 741-758, 2017.

[16] G. Mountrakis, J. Im, and C. Ogole, "Support vector machines in remote sensing: A review, " ISPRS Journal of Photogrammetry and Remote Sensing, vol. 66, pp. 247-259, 2011.

[17] U. Maulik, and D. Chakraborty, "Remote Sensing Image Classification: A survey of support-vector-machine-based advanced techniques, “ IEEE Geoscience and Remote Sensing Magazine, vol. 5, pp. 33-52, 2017.

[18] X. Yang, "Parameterizing Support Vector Machines for Land Cover Classification," Photogrammetric Engineering and Remote Sensing, vol. 77, no. 1, pp. 27-37, 2011. 